\title{
Observation of Ultrafast Solid-Density Plasma Dynamics Using Femtosecond X-Ray Pulses from a Free-Electron Laser
}

\author{
Thomas Kluge, ${ }^{1, *}$ Melanie Rödel,,${ }^{1,2}$ Josefine Metzkes-Ng, ${ }^{1}$ Alexander Pelka, ${ }^{1}$ Alejandro Laso Garcia, ${ }^{1}$ Irene Prencipe,${ }^{1}$ \\ Martin Rehwald, ${ }^{1,2}$ Motoaki Nakatsutsumi, ${ }^{3}$ Emma E. McBride, ${ }^{3,4}$ Tommy Schönherr, ${ }^{1,2}$ Marco Garten, ${ }^{1,2}$ \\ Nicholas J. Hartley, ${ }^{1,8}$ Malte Zacharias, ${ }^{1,2}$ Jörg Grenzer, ${ }^{1}$ Artur Erbe, ${ }^{1}$ Yordan M. Georgiev, ${ }^{1, \dagger}$ Eric Galtier, ${ }^{4}$ \\ Inhyuk Nam, ${ }^{4}$ Hae Ja Lee, ${ }^{4}$ Siegfried Glenzer, ${ }^{4}$ Michael Bussmann, ${ }^{1}$ Christian Gutt, ${ }^{5}$ Karl Zeil, ${ }^{1}$ Christian Rödel, ${ }^{4,6}$ \\ Uwe Hübner, ${ }^{7}$ Ulrich Schramm, ${ }^{1,2}$ and Thomas E. Cowan ${ }^{1,2}$ \\ ${ }^{1}$ Helmholtz-Zentrum Dresden-Rossendorf, Bautzner Landstraße 400, 01328, Dresden, Germany \\ ${ }^{2}$ Technical University Dresden, 01069 Dresden, Germany \\ ${ }^{3}$ European XFEL, Holzkoppel 4, 22869 Schenefeld, Germany \\ ${ }^{4}$ SLAC National Accelerator Laboratory, 2575 Sand Hill Road, Menlo Park, California 94025, USA \\ ${ }^{5}$ Universität Siegen, Adolf-Reichwein-Straße 2, 57068 Siegen, Germany \\ ${ }^{6}$ Freidrich-Schiller-Universität, Max-Wien-Platz 1, 07743 Jena, Germany \\ ${ }^{7}$ Leibniz Institute of Photonic Technology, Albert-Einstein-Straße 9, 07745 Jena, Germany \\ ${ }^{8}$ Open and Transdisciplinary Research Institute, Osaka University, Suita, Osaka 565-0871, Japan
}

(Received 2 January 2018; revised manuscript received 7 June 2018; published 13 September 2018)

The complex physics of the interaction between short-pulse ultrahigh-intensity lasers and solids is so far difficult to access experimentally, and the development of compact laser-based next-generation secondary radiation sources, e.g., for tumor therapy, laboratory astrophysics, and fusion, is hindered by the lack of diagnostic capabilities to probe the complex electron dynamics and competing instabilities. At present, the fundamental plasma dynamics that occur at the nanometer and femtosecond scales during the laser-solid interaction can only be elucidated by simulations. Here we show experimentally that small-angle x-ray scattering of femtosecond $\mathrm{x}$-ray free-electron laser pulses facilitates new capabilities for direct in situ characterization of intense short-pulse laser-plasma interactions at solid density that allows simultaneous nanometer spatial and femtosecond temporal resolution, directly verifying numerical simulations of the electron density dynamics during the short-pulse high-intensity laser irradiation of a solid density target. For laser-driven grating targets, we measure the solid density plasma expansion and observe the generation of a transient grating structure in front of the preinscribed grating, due to plasma expansion. The density maxima are interleaved, forming a double frequency grating in $\mathrm{x}$-ray free-electron laser projection for a short time, which is a hitherto unknown effect. We expect that our results will pave the way for novel time-resolved studies, guiding the development of future laser-driven particle and photon sources from solid targets.

DOI: 10.1103/PhysRevX.8.031068

Subject Areas: Plasma Physics

\section{INTRODUCTION}

The solid-density plasmas created in the interaction of an ultrashort, ultrahigh-intensity (UHI) laser pulse with a solid target are a source of femtosecond, high-charge electron [6] and ion bunches [7-10], extreme ultraviolet (XUV) radiation [11-13], and neutrons [14], making them promising candidates for future particle accelerators or radiation

\footnotetext{
t.kluge@hzdr.de

${ }^{\dagger}$ On leave from: Institute of Electronics, Bulgarian Academy of Sciences, Sofia, Bulgaria.

Published by the American Physical Society under the terms of the Creative Commons Attribution 4.0 International license. Further distribution of this work must maintain attribution to the author(s) and the published article's title, journal citation, and DOI.
}

sources, e.g., for tumor therapy [1,2], laboratory-astrophysics [3,4], and fusion [5]. The fundamental property of such ultraintense lasers is the acceleration of plasma electrons to relativistic energies within a single laser period, i.e., less than a few femtoseconds in the case of optical or nearinfrared UHI lasers. Until now a fundamental impediment of the ongoing research of UHI laser-solid interactions has been the limited experimental capability of diagnosing the arising basic processes during the laser interaction on the relevant scales that range from subfemtosecond to hundreds of femtoseconds and from few nanometers to few hundred nanometers. Some of the most important physical processes are, for example, the generation of plasma oscillations [15] and plasma waves [16], transport of relativistic electrons and plasma heating [17,18], instability development [16,19-24], and the generation of strong magnetic fields 
due to the strong currents generated by the laser pressure [17]. A fundamental process is the expansion of the irradiated plasma into vacuum [25-27] during the laser interaction, governing the surface dynamics and laser absorption both prior to and during the laser main pulse.

For each of the aforementioned applications a correspondingly tailored surface structure can enhance laser absorption and interaction, electron acceleration, and hence all subsequent processes. In fact, it has been shown that a preplasma density gradient, e.g., generated by laser intensity prior to the main pulse, strongly affects absorption [28] and the generation of secondary radiation such as proton acceleration [29] and XUV radiation [30]. Grating targets have even shown nearly complete absorption [31], resulting in enhanced ion acceleration [32] and XUV generation [33].

Measuring, controlling, and eventually optimizing collective particle motion excited by, and interleaved with, the intense laser light is the key to pushing the development of laser-based radiation sources further. Conventionally, these dynamic effects can only be accessed by numerical simulations, indirect experimental methods (e.g., particle or radiation emission from the bulk), or optical methods $[34,35]$, which are, however, all limited in either spatial or temporal resolution or to the optically transparent lowdensity regions.

The present work aims at making the few femtosecond, few nanometer regime directly accessible at solid density and relativistically intense laser irradiation by employing small-angle $\mathrm{x}$-ray scattering (SAXS) using $\mathrm{x}$-ray freeelectron laser (XFEL) pulses [36]. With the advent of XFELs there have been many approaches to use them as a probe for UHI laser-driven solid-density plasmas. X-ray beams can penetrate solid-density plasmas and hence can resolve the dynamics at high densities throughout the whole target. Taking advantage of the unique properties of XFEL pulses, e.g., their short duration, high number of photons per bunch, high transverse coherence, monochromaticity, and polarization, the XFEL can widely expand the capabilities of established diagnostic tools. While x-ray diffraction of hard XFEL beams [37,38] typically provides a resolution on the order of angstroms, direct imaging methods using parallel beams are limited mainly by the detector resolution, which typically is on the order of microns [39], whereas for the method of magnified $\mathrm{x}$-ray phase contrast imaging, using a strongly focused beam, the resolution is limited by the $\mathrm{x}$-ray spot size, which is constrained by the SASE bandwidth to at least a few hundred nanometers [40-42]. For seeded XFEL beams the focus could be much smaller, but the imaging quality is eventually limited by the flux due to the small elastic cross section.

SAXS can bridge the gap with a resolution between the nanometer and few micron scale. It is an established x-ray scattering technique [43] which employs the fact that for small scattering angles the relative path differences of photons scattered at neighboring electrons (separated by $\boldsymbol{r}$ ) can be linearized to $\Delta \varphi=-\boldsymbol{r} \cdot \boldsymbol{q}$, and, hence, the resulting scattering pattern summing up all scattered photons $\propto\left|\int n_{e}(\boldsymbol{r}) e^{-r q} d \boldsymbol{r}\right|^{2}$ is given simply by the absolute square of the Fourier transform (FT) of the electron density distribution. On XFELs, it has been developed in recent years starting with a demonstration of the principle that with an ultrashort $\mathrm{x}$-ray pulse a scattering pattern can be recorded before destruction [44], followed by the first XFEL pump, XFEL probe scattering experiments demonstrating both femtosecond and nanometer resolution [45]. It took almost another 10 years to perform the first optical pump, XFEL probe study with similar resolutions, with an optical pump laser intensity on the order of $10^{15} \mathrm{~W} / \mathrm{cm}^{2}$ [46].

On the path towards ultraintense, ultrarelativistic lasersolid interactions that will be possible at European XFEL and SACLA in the near future [47], here we report on a scattering experiment with the currently most intense optical pump laser available at an XFEL, the short-pulse highintensity (HI) laser at the Matter in Extreme Conditions (MEC) end station of LCLS at SLAC [48,49]. We present the first measurement of the spatial density distribution of a high-intensity (near-relativistic) short-pulse laser-driven solid-density plasma to the nanometer level, with femtosecond resolution. SAXS, using XFEL pulses, allows for a single-shot direct electron density measurement to study in situ the plasma expansion and nonlinear dynamics before, during, and directly after the laser irradiation. Employing the pump-probe technique, we can follow the expansion of the plasma at solid density in real time and compare it directly to simulations.

\section{EXPERIMENTAL SETUP}

The experiments were performed at the MEC end station at LCLS with the Ti:sapphire near-infrared HI short-pulse laser. The pulse duration was $83 \mathrm{fs}$ and the spot size was $16 \mu \mathrm{m} \times 30 \mu \mathrm{m}$, both at FWHM. We performed shots at two laser pulse energies, $1 \mathrm{~J}$ corresponding to $5 \times$ $10^{17} \mathrm{~W} / \mathrm{cm}^{2}$ for most of the shots (all of the systematic pump-probe results shown below in Figs. 2 and 3), and approximately $400 \mathrm{~mJ}$ for a few single shots (including the one shown in Fig. 4). The XFEL beam had a diameter of 5-10 $\mu \mathrm{m}$ and pulse duration of $40 \mathrm{fs}$, both at FWHM. The photon energy was $8 \mathrm{keV}$, with approximately $N_{0}=10^{11}$ photons per pulse (the exact number varies from shot to shot due to different absorbers). Synchronization with the HI short-pulse laser and LCLS XFEL beam was achieved to an accuracy of $120 \mathrm{fs}$, measured by a spatially encoding autocorrelator [50,51] (for more details about the optical setup, see Fig. 1 and Sec. V). Our samples were $2-\mu \mathrm{m}$-thick silicon membranes with an inscribed surface grating. Additionally to being a fundamentally interesting target for optimizing the laser-solid interaction applications, the advantage of using tailored grating surface membranes is 
the large increase in scattering signal compared to a flat surface. We can now follow small variations in the scattering signal to detect few nanometer structural changes, induced by the laser irradiation, with a high spatial resolution reaching down to a nanometer and below.

In the following, we present a series of shots on surface gratings with periods $g$ ranging from 150 to $500 \mathrm{~nm}$ where the ridge widths $b$ were approximately half of the grating period and the depth $100 \mathrm{~nm}$. The rectangular ridges and the membrane itself were flat to the level of $\sigma_{0}=(5.4 \pm 0.7) \mathrm{nm}$, which was measured for each target by two consecutive XFEL-only SAXS measurements at reduced intensity that did not damage the target ("preshots") [52] (see Sec. V). Those grating dimensions, besides being in the relevant range for absorptionenhancing surface structures, are also in the range of the spatial dimensions of instabilities in relativistically driven plasmas and are therefore also interesting on a technical level for future applications of SAXS. For example, among others, Rayleigh-Taylor-like instabilities leading to a surface rippling at relativistic intensities [16,23], two-stream instabilities in the target [22] or at the target rear surface [24], and Weibel-like instabilities occurring during the plasma propagation in vacuum after laser acceleration [53] lead to a complex interplay that could be studied by the SAXS method [36,54]. For example, in the case of the Rayleigh-Taylor instability, the laser light pressure pushes against the reflective solid-density plasma front, leading to a growth of initially small density fluctuations to form ripples even on an initially flat target surface and eventually to characteristic fingerlike plasma modulations. While the optical laser intensity used in the present experiment is not quite sufficient to drive those instabilities, based on the current understanding the spatial extent of those modulations is expected to be on the order of a few tens of nanometers or more, which is comparable to the dimensions of our front side grating structure.

In the regime relevant for this work, the dominant source of small-angle X-ray scattering is coherent elastic Thomson scattering from electrons. In the SAXS geometry, the scattering pattern is obtained in kinetic and Born approximations by the absolute square of the exit wave FT [43]. With an appropriate model for the density in real space, the gradient of the expanded plasma and other spatial features can be characterized by fitting the respective correlation function to the scattering pattern in reciprocal space [54].

\section{RESULTS}

Typical scattering patterns are shown on the right-hand side of Fig. 1 for three different HI-laser delay times, with their profiles along the horizontal direction analyzed in Fig. 2. The scattering signal covers the whole field of view of the detector, with decreasing intensity at larger scattering vector values $q \equiv\left(2 \pi / \lambda_{X}\right) 2 \sin (\Theta / 2) \quad\left(\lambda_{X}\right.$ is the XFEL wavelength and $\Theta$ is the scattering angle). With increasing delay time we observe a decrease of the scattering signal at large $q$ values. We interpret this as an exponential roll-off due to the expansion of the plasma bulk, in analogy to the Debye-Waller factor.

We model the plasma line density along the grating direction as the convolution of a grating delta comb, a single slit (ridge), and the ridge edge shape described by a Gaussian, $\left(N_{b} / b\right) \exp \left(-x^{2} / 2 \sigma^{2}\right)$. The density along a ridge edge is then
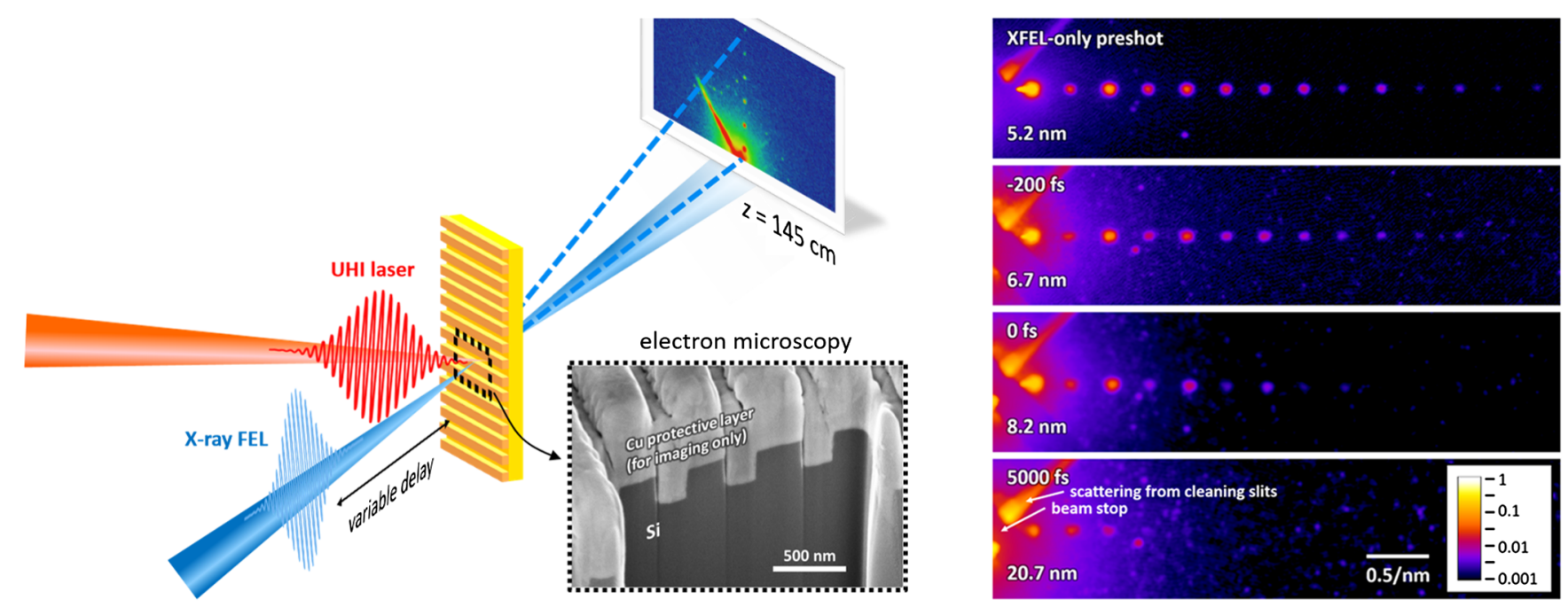

FIG. 1. Schematic of the SAXS experimental setup. Left: The HI laser pulse is focused on a silicon grating target under $45^{\circ}$ parallel to the grating ridges and leading to a high-intensity laser-plasma interaction. The XFEL pulses probe the laser-plasma dynamics under $90^{\circ}$. The inset shows an electron scanning microscopy image of a representative target sample with grating period $500 \mathrm{~nm}$ (targets were covered with $\mathrm{Cu}$ only for imaging). Right: Examples of scattering patterns of a grating with period $g \cong 300 \mathrm{~nm}$ and ridge width $b \cong 150 \mathrm{~nm}$ for different XFEL delays (rotated by $90^{\circ}$, normalized to the respective intensity in the second scattering peak-the first one was sometimes oversaturated). 

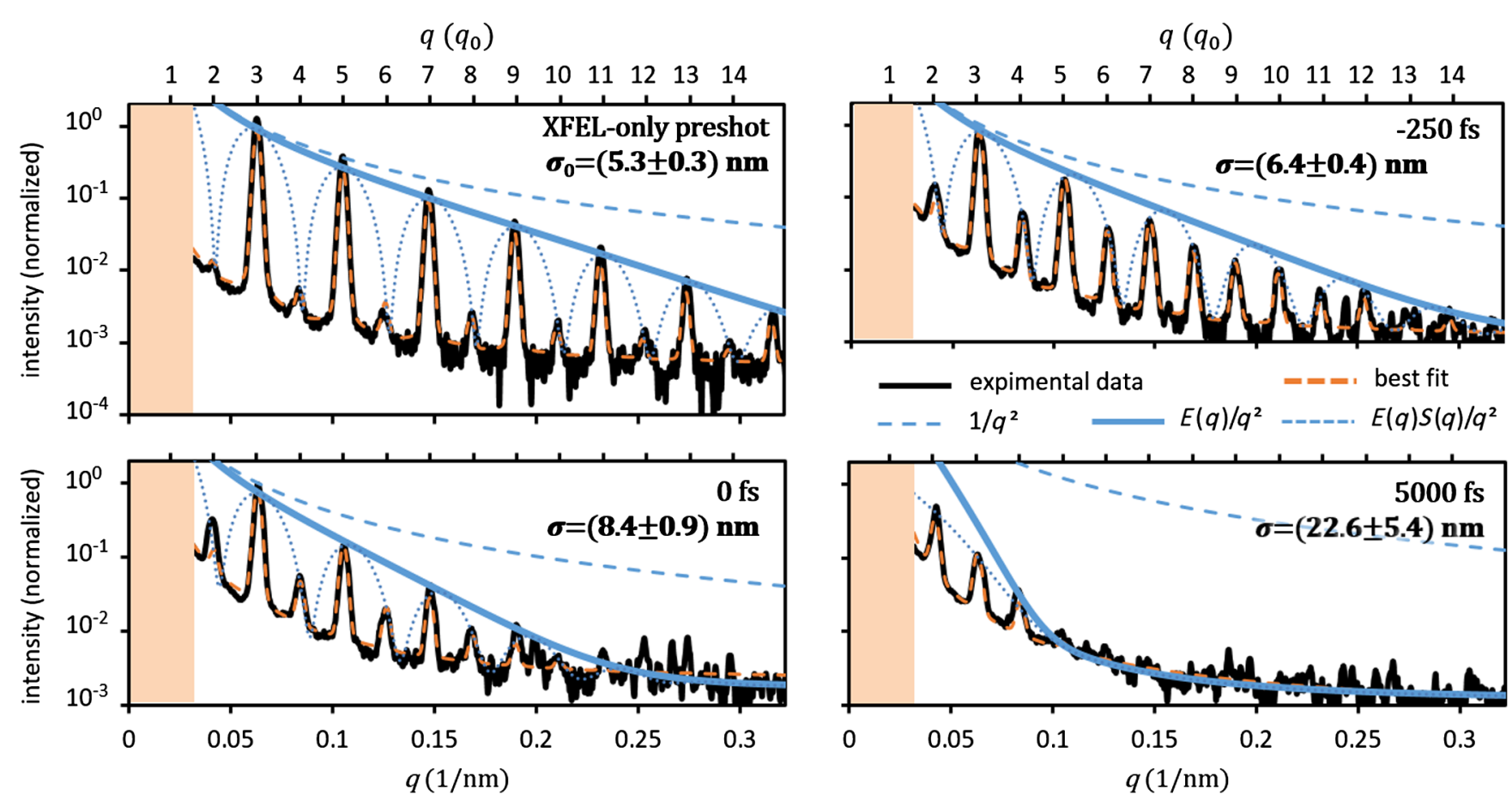

FIG. 2. Lineouts and fitting. Cuts through the horizontal direction through the scattering peaks of the SAXS pattern for the four shots shown in the right-hand side of Fig. 1 (black lines) and fitting results. From the full fits (dashed orange lines) the individual contributing terms of Eq. (1) are shown: $1 / q^{2}$ corresponds to scattering from a perfectly sharp step (blue long dashed lines); $E(q) / q^{2}$ from a smooth edge with expansion parameter $\sigma$ (blue solid lines); and $S(q) E(q) / q^{2}$ from a single slit with smooth edges (blue dotted lines). Orange area marks the region with oversaturated pixels and was not used for fitting.

given by an error function, $\tilde{n}_{e} \equiv\left(N_{b} / 2 b\right)[\operatorname{erf}(x / \sqrt{2} \sigma)+1]$, where $N_{b}$ is the total number of electrons in a ridge in the illuminated area. The expansion parameter $\sigma$ defines the smoothness of the grating ridge and is therefore a measure of the plasma expansion scale length.

The scattered intensity in the distance of a unit length is derived from the product of the respective squares of the normalized FT of the grating $G(q)$, single slit $S(q)$ [55], and error function $E(q)$ [56],

$$
I(q)=\frac{I_{0}}{q^{2}} \cdot G(q) \cdot S(q) \cdot E(q),
$$

with

$$
\begin{gathered}
I_{0}=I_{X} r_{e}^{2}\left(2 N \frac{N_{b}}{b}\right)^{2}, \\
G(q)=\left[\frac{\sin (N q g / 2)}{N \sin (q g / 2)}\right]^{2}, \\
S(q)=\sin ^{2}\left(\frac{q b}{2}\right), \\
E(q)=e^{-q^{2} \sigma^{2}}
\end{gathered}
$$

( $I_{X}$ is XFEL intensity, $r_{e}$ is classical electron radius, and $N$ is the number of ridges in the illuminated area).
In Fig. 2, we illustrate the quantitative determination of the time-dependent plasma expansion by describing the data using Eq. (1) as an estimator. The single slit scattering envelope $S(q)$ from Eq. (4) causes some peaks described by the grating spatial frequency harmonics in $G(q)$ to be suppressed due to the phase matching of $S(q)$ and $G(q)$ related to the $b / g$ ratio; i.e., slight geometric variances between targets (and between the XFEL-only preshot and the HI laser-driven shot) lead to more or less extinguished even peaks. Finally, the edge shape envelope $E(q)$ from Eq. (5) yields the expansion parameter $\sigma$ describing the bulk expansion.

We performed a multiparameter fit including all variables to infer $\sigma$ from the best fit. The full fitting procedure is described in Sec. V. The fitting results for all of our fullintensity laser shots are summarized in Fig. 3, together with particle-in-cell simulation results (orange line; see Sec. V). When the laser pulse hits the target, a fast expansion of the grating ridges is observed; i.e., the value of $\sigma-\sigma_{0}$ increases. The agreement between the measurement and the simulation is excellent: both the fast expansion during the femtosecond optical laser pulse irradiation and the stagnation thereafter are well reproduced. We note that despite the laser contrast showing significant prepulses approximately $50-40$ ps prior to the main pulse (see Sec. V for details on the temporal contrast), an expansion of the target bulk prior to the optical laser main pulse was not observed, on the 


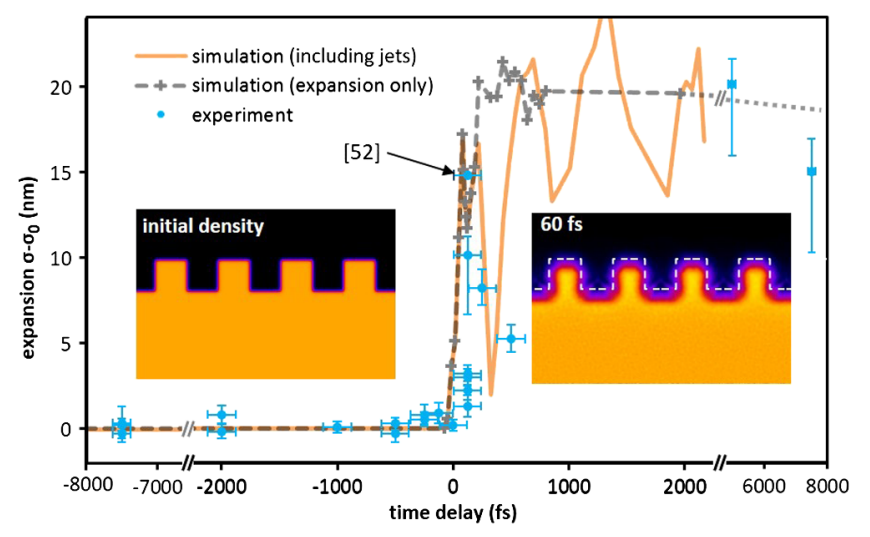

FIG. 3. Surface expansion into vacuum. Expansion parameter $\sigma-\sigma_{0}$ as a function of XFEL delay extracted from the scattering patterns of all our shots with HI laser energy of $1 \mathrm{~J}$ (blue circles). Orange solid (gray dashed) line shows $\sigma$ extracted from the simulation by fitting a grating with error-function-shaped edges to the density integrated through the target, including (excluding) the plasma jets emitted between the grating ridges. The dotted line is the extrapolation of the dashed line beyond the simulated delay time of $2 \mathrm{ps}$. Excluding the jets, $\sigma$ resembles the expansion of the ridges, while including them $\sigma$ corresponds to the shape of the plasma jets when their density dominates over that of the remaining preinscribed ridges at delays larger than $\approx 270$ fs (see main text for details). The insets show the plasma density from simulation (color scale same as in Fig. 4).

scale of the nanometer to tens of nanometers sensitivity of our setup-proving ultrashort XFEL scattering before destruction.

We can extract the expansion velocity by linear interpolation during the expansion phase around $t=0$. With the timing accuracy limited by the XFEL delay jitter, we can estimate only a lower limit for the expansion velocity of approx. $0.04 \mathrm{~nm} / \mathrm{fs}$, which compares to $0.19 \mathrm{~nm} / \mathrm{fs}$ estimated in the simulation. From the simulation we find that after approximately $100 \mathrm{fs}$ the expansion has progressed so far that the rising and falling edges of the ridges intersect and penetrate each other. We infer from the simulation that the laser pushes the target surface-and even more the ridges - forward into the bulk, so that the ridges eventually even disappear after approximately 200 fs and only remain as density modulations in the compressed density layer (e.g., see the video in Supplemental Material [57] or Fig. 4, top). Their edge shape quickly stagnates at an error function width of approximately $25 \mathrm{~nm}$ as the driving charge separation neutralizes after the optical laser pulse has passed; $\sigma$ then remains almost constant over the remaining $1.5 \mathrm{ps}$ of the simulation, and likely it does also beyond. This observation is in agreement with the experiment, confirming that the compressed front surface layer indeed remains intact over the time course of a few picoseconds.

We observe an interesting effect in the simulations of the expanding plasma grating that has not been described so far. While two neighboring grating ridges expand and form
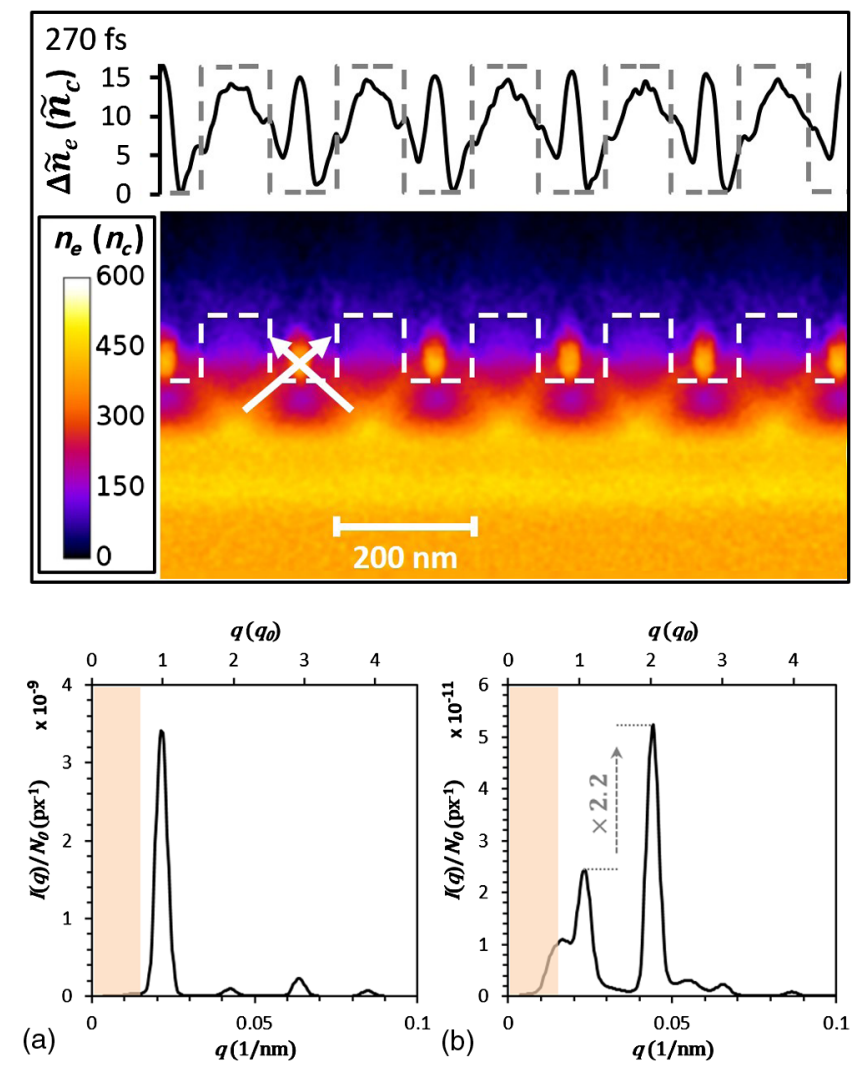

FIG. 4. Transient plasma grating. Top: Particle-in-cell simulated electron densities (in units of $n_{c}=1.7 \times 10^{21} \mathrm{~cm}^{-3}$ ) suggest the emission of plasma jets (white arrows) from the sharp edges at the surface. Integrating along the vertical direction (top panel), we find the grating frequency doubled. Bottom: Cuts through the experimental scattering pattern for an XFEL-only preshot (a) and main shot with HI laser energy $400 \mathrm{~mJ}$ and delay 6 ps (b). In the main shot we observe the intensity of the second peak being larger than that of the first peak, being forbidden for a single grating with grating vector $q_{0}$. See Supplemental Material for details [57].

plasma jets, they intersect so that an additional plasma grating is generated at the intermediate position; see Fig. 4 and the movie in Supplemental Material [57]. In the simulation this happens for the first time around $270 \mathrm{fs}$ after the main pulse, and repeats periodically after approximately a picosecond. When the jets cross each other, the density modulation can get larger than that of the expanding ridge remainders, then dominating the measurements of the expansion parameter. This leads to the oscillating structure in the expansion parameter extracted from the simulation (Fig. 3, solid orange line). For a short time of a few tens of femtoseconds the density of the transient grating can match the preinscribed grating. In the projection integrated along the XFEL, both together then form a modulation of twice the initial grating frequency (see Fig. 4, top).

Since the SAXS signal is sensitive to the spatial frequencies, we can study this novel effect by analyzing the heights and extinction of the individual peaks in the 
scattering patterns. For the ideal grating, with $b / g \approx 1 / 2$, $S(q)$ minimizes near the position of the even harmonics of the grating spatial frequency $q_{0}$ (compare dashed line in Fig. 2). The fabricated gratings slightly deviate from the ideal $b / g$ ratio, so that the exact peak heights vary between individual samples and also even harmonics may appear; for $b / g>1 / 2$, the first minimum of $S(q)$ shifts to larger $q$ and vice versa. This means that upon optical laser irradiation, when the grating ridge expands, the second harmonic peak at $q=2 q_{0}$ can increase or decrease relative to the first peak, depending on the initial position of the single slit minimum. The maximum height of the second peak is, however, limited to the height of the envelope (see Supplemental Material [57]).

However, in one shot we observe a peculiar excess scattering intensity in the second peak; i.e., $I\left(2 q_{0}\right)$ is more than twice larger than $I\left(q_{0}\right)$ (see Fig. 4, bottom). The most likely explanation for the second peak being larger than the first one is the existence of a structure of twice the preinscribed grating frequency in the target density projected along the XFEL direction-lending evidence that the SAXS method is sensitive also to such detailed dynamics and could be used to optimize the generation of transient nanograting structures (see Supplemental Material for details [57]). Especially in the context of other mechanisms employing or leading to transient gratings this may become relevant, considering, e.g., four-wave mixing in transient gratings [58], the power distribution in holraums [59], or harmonic generation [13] where SAXS could be used for sub-lasercycle diagnostics of the laser-plasma interaction [54].

In the present case the double grating exists only for a few tens of femtoseconds, acting as a fast switch for the x-ray beam in the direction of the second maximum. This explains why we observe excess intensity only in one shot: The timing of the XFEL probe arrival must match the formation of the transient grating quite accurately, so in the majority of shots we would likely miss it. The statistics of this study was mainly limited by the time needed to position the target in the interaction point. The hard limitation would be set by the repetition rate of the optical pump laser, which is typically on the order of $10 \mathrm{~Hz}$, which would allow a much more detailed study if the target delivery and alignment procedures could be automated [60].

\section{DISCUSSION AND OUTLOOK}

In summary, we present the first quantitative measurement of plasma expansion into vacuum [25] at solid density with nanometer, few-femtosecond resolution, driven by a near-relativistic high-intensity laser. It is well known that the critical density surface is expanded by a significant fraction of the laser wavelength, but how much the soliddensity surface expands was not well known before this study. We have experimentally verified that the surface expands but, besides this, stays intact during the interaction and a few picoseconds beyond (for the intensity and pulse duration regime we examined). While information of the critical density surface is important for laser absorption, information of the solid-density dynamics is crucial for understanding complex processes, e.g., laser-generated particle transport, bulk heating, and instability development, as well as production of static electric and magnetic fields that are generated strongly at the steep density jump (resistivity jump).

The concept of preinscribed gratings serving as a reference and providing a strong signal at large $q$ from a well-defined spatial region was the key for the high spatial sensitivity. Placing the gratings at the rear surface would immediately allow us to characterize the TNSA process $[61,62]$, where it would enable the measurement of the solid-density plasma expansion on the relevant scales of few nanometers and femtoseconds. The grating method could further be extended, for example, to buried gratings for studying isochoric or buried layer heating [63,64], gratings of a specific material with bound-bound resonances at the XFEL energy for resonant scattering [65], or more complex 2D structures-e.g., to encode spatial markers into the $q$ space. For example, a thin layer of a high-density material within a low-density material could serve as a spatial limiter for density-dependent features such as plasma oscillations, and could help mitigate the problem of plasma motion blur or other volume effects.

Likewise, it might be interesting for future applications such as femtosecond $\mathrm{x}$-ray manipulation or switching to vary the detailed grating geometry (e.g., chirped gratings, inclined gratings) in order to tailor the characteristics of the transient gratings.

It is important to point out that in the present SAXS geometry we measure the integrated density through the target along the XFEL beam direction, i.e., including both effects on the surface and in the bulk Si substrate, which on the picosecond timescale might undergo some compression or structural changes from the laser. Introducing volumetrically structured multicomponent targets could also help to separate surface and bulk effects.

At higher laser intensities that will become available soon at European XFEL, SACLA, and possibly MEC, where, for example, relativistic instabilities are expected to lead to spatial modulations of tens of nanometer to microns scale within a few femtoseconds, SAXS measurements might help to optimize future laser-based particle and radiation sources and therefore will pave the way for novel applications.

\section{METHODS}

\section{A. Optical laser}

The optical laser used for target excitation, i.e., plasma generation, is the Ti:sapphire-based high-power laser system at the MEC end station at LCLS. The laser system provides ultrashort pulses ( $\tau=80 \mathrm{fs}$ ) at a central wavelength of $800 \mathrm{~nm}$, the pulses containing an energy of $1 \mathrm{~J}$ before the compressor and $460 \mathrm{~mJ}$ on target. The laser pulse 


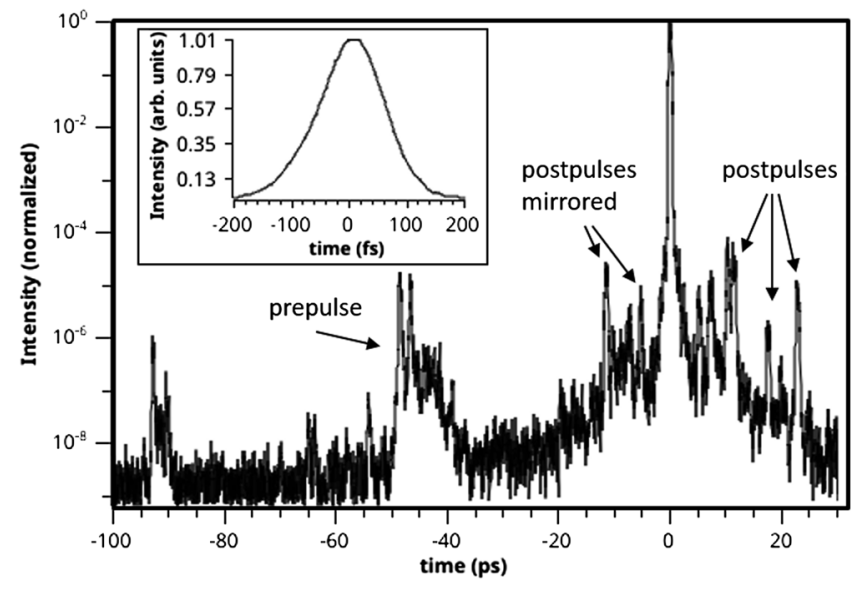

FIG. 5. Laser contrast and pulse duration. The laser contrast base level was measured by a cross-correlator and is better than $10^{-8}$. This suppresses the laser intensity below the ionization threshold of Si until the prepulse at $-50 \mathrm{ps}$. The main laser pulse temporal shape is shown in the inset by the autocorrelation trace.

was focused by an off-axis parabolic mirror, the focal spot size $(30 \mu \mathrm{m} \times 16 \mu \mathrm{m}$ FWHM) was measured as the average of 26 single-shot measurements (systematic and statistical error below 2\%). Within the FWHM of the spot, $22 \%$ of the laser pulse energy is contained, leading to an optical laser intensity (average over FWHM area) of $4 \times 10^{17} \mathrm{~W} / \mathrm{cm}^{2}$, which for Gaussian shape corresponds to a peak intensity $5 \times 10^{17} \mathrm{~W} / \mathrm{cm}^{2}$. The intensity on target taking into account the angle of incidence of $45 \mathrm{deg}$ is a factor of $\sqrt{1 / 2}$ smaller. The temporal intensity contrast of the laser pulse-i.e., the ratio of the background laser intensity normalized to the peak intensity of the ultrashort pulse-was characterized with a third-order cross-correlator [66], with a temporal resolution of approximately 100 fs; see Fig. 5. The detection threshold lies at $\approx 2 \times 10^{-9}$. The prepulse at $-48 \mathrm{ps}$ is real according to the measurement, whereas other pulses occurring at negative delay (i.e., before the main pulse) could be artifacts from the measurement itself. As was shown in Fig. 3, the prpulses do not lead to a measurable expansion of the sample. However, this does not rule out the possibility of formation of a low-density preplasma, which, however, would have to have either a density small enough to not be detected or a scale length large enough that the exponential roll-off occurs well before the first scattering order.

\section{B. XFEL}

The LCLS beam fundamental was focused with compound refractive lenses into the MEC experimental area to a spot size between 5 and $10 \mu \mathrm{m}$. The third harmonic was only weakly focused and hence its intensity is greatly reduced on target. The XFEL pulse was then attenuated by various $\mathrm{Si}$ and $\mathrm{Cu}$ absorbers to ensure the scattering signal was within the dynamic range of the PIXIS XF 2048B camera. For most of the XFEL-only preshots the XFEL transmission was $\approx 1 \%$ (10\% Si and $13 \% \mathrm{Cu}$ transmission), for the main shots $\approx 5 \%$ (50\% $\mathrm{Si}$ and $13 \% \mathrm{Cu}$ transmission), i.e., $5 \times 10^{10}$ photons/pulse. The absolute number of photons was determined for each shot by the three calibrated MEC gas detectors. The direct XFEL beam transmitted through the target was blocked by a beam-block wire in front of the vacuum exit window. We recorded two XFELonly preshots before each optical laser shot-the first to qualify the undriven target structure and the second to prove that the XFEL at the selected intensity does not damage the sample.

\section{Synchronization}

A crucial point of the experimental setup and preparation is the spatial and temporal overlap of the optical and XFEL pulse as well as the target at the interaction point. The interaction point is fixed in space via two independent high-resolution alignment imaging systems $(\approx 0.2 \mu \mathrm{m} /$ pixel resolution at a field of view of $200 \times$ $360 \mu \mathrm{m}^{2}$ and $290 \times 390 \mu \mathrm{m}^{2}$, respectively). For the spatial alignment of the XFEL pulse, a target (500- $\mu$ m-thick Si wafer) is placed in the interaction plane and a burst of XFEL pulses is used to create an $\approx 30 \mu$ m diameter damage (hole) in the target, which can be imaged with the alignment imaging systems. This defines the interaction point in the interaction plane. The optical pulse as well as the target are then aligned to this interaction point using the alignment imaging systems. The overlap of the XFEL pulse with the grating structure was independently confirmed in the nondestructive preshots taken before every combined irradiation of the target by the optical laser and XFEL.

The temporal overlap of the optical and XFEL pulse at the interaction point is measured with a $20-\mu$ m-thick YAG plate. The XFEL pumps the crystal in target position by generating free carriers in the YAG and therefore changing its refractive index. This change in refractive index can be probed by the optical laser to determine the synchronization point. This setup is comparable to the spatial encoding time tool presented in Refs. [50,51]. The same principle is applied in the time tool installed in the XFEL beam path upstream of the interaction point in order to monitor the shot-to-shot timing jitter between the optical and XFEL pulse. Here, the ultrafast free-carrier generation in a $\mathrm{Si}_{3} \mathrm{~N}_{4}$ slab is probed by a pulse split from the main optical pulse. The time tool measured a timing jitter for the relative arrival time between the optical and XFEL pulse 119 fs, which determines the delay time error bars in Fig. 3.

\section{Small-angle x-ray scattering}

Depending on the $\mathrm{x}$-ray photon energy and the HI laser intensity, and therefore ionization degree and opacity of the plasma, the scattering of $\mathrm{keV}$ x-ray photons is dominated by elastic or inelastic Thomson scattering or 
photoionization process [65]. Here we assume that the XFEL photon energy is far enough from bound-bound transition energies so that excitations and ionization of inner electronic shells of atoms or ions by the XFEL can be ignored. For small scattering angles the signal is then dominated by elastic Thomson scattering.

In the SAXS geometry the scattering image is obtained in Born approximation simply by absolute square of the exit wave FT [43], the latter being given by the product of the plasma electron density integrated along the x-ray propagation direction and the Gaussian-like illumination. The respective spatial and temporal resolution are fundamentally limited only by the x-ray wavelength and pulse duration-enabling nanometer and few femtosecond resolution. Of course, for a real experiment, for example, the quality of the spatial XFEL beam profile and timing jitter may reduce the resolution. We used a PIXIS XF 2048B $\mathrm{x}$-ray camera to record the scattering pattern. For absolute photon numbers we calibrated it using an $\mathrm{Am}^{241}$ and an $\mathrm{Fe}^{55}$ source. The system resolution is dictated by the PIXIS point spread function and XFEL beam divergence, which are both between 2 and 3 pixels on the detector.

\section{E. Fitting procedure and nanometer resolution}

Though great care was taken during the target fabrication process to produce as sharp and regular gratings as possible, the gratings cannot provide an exact aspect ratio ridge width $b$ to grating period $g$ of exactly $1 / 2$ due to technological limits; see inset in Figs. 1 and 6. Therefore, it cannot be assumed that every even peak is extinct and every odd one is completely unaffected. Thus we cannot simply fit the edge scattering function (FT of the error function) to the scattering peak values. Rather, we must fit the full grating scattering equation [Eq. (1)]. We fixed the number of illuminated grating ridges $N$ to the respective calculated values in the XFEL FWHM spot-neglecting the shape of the XFEL intensity radial profile-since it has almost no effect on values of the fitted width $\sigma$, and the point spread function of the detector is slightly larger than the FT of the illumination spot. The error function model for the density distribution was chosen based on the simulations (see below). However, as can be shown, for many other choices of the ridge edge shape the fit does not change significantly. In the fitting algorithm we also take into account a finite detector point spread function, which broadens and hence reduces the peak signal (in Fig. 7 we scaled the envelopes accordingly) and completely washes out the grating scattering side maxima. With the present detector it would only be possible to sample the fine side maxima of the grating function if an almost parallel x-ray beam was used (so that the divergence is significantly smaller than the peak distance to resolve) and the distance between the target and detector was increased to approximately $5 \mathrm{~m}$, which is technically not feasible due to space limitations and would reduce the angular angle covered by the detector drastically.
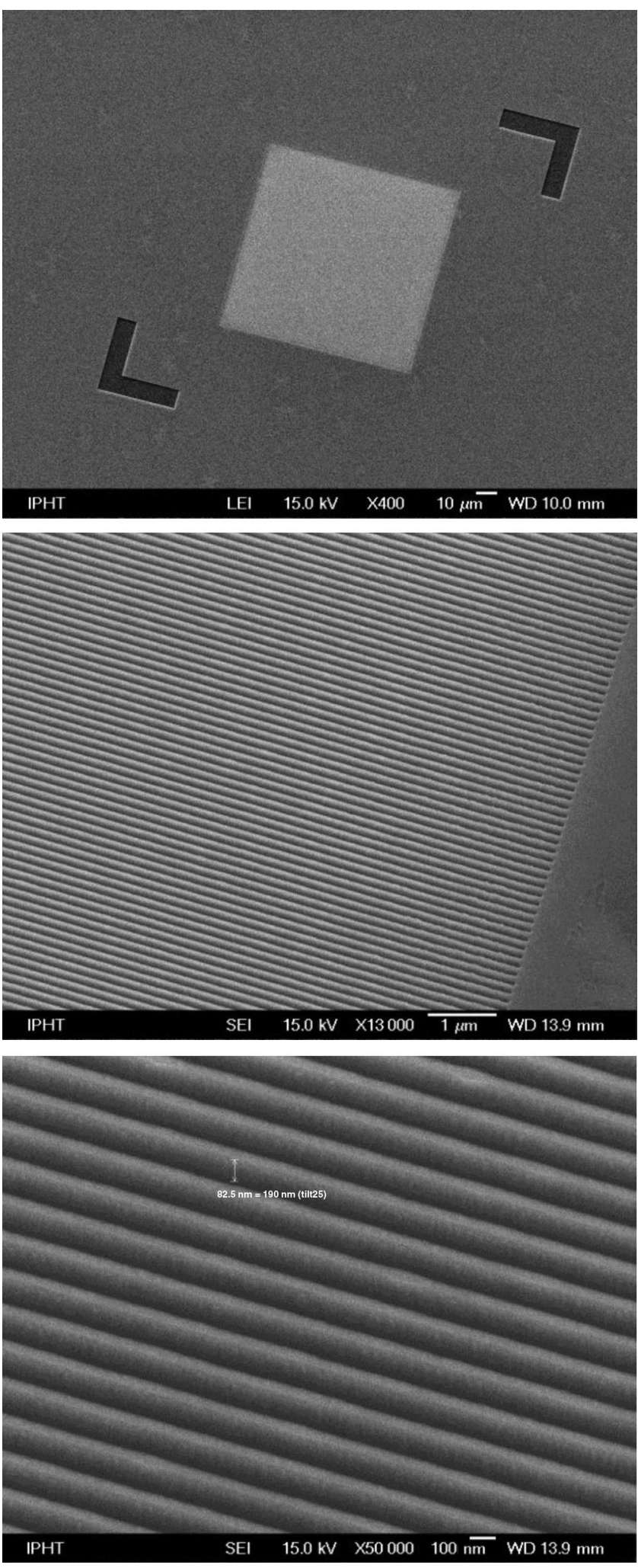

FIG. 6. Electron microscope images of a sample target $(g=200 \mathrm{~nm})$ at three different zoom levels.

Since the large scattering angles carry the information of small spatial scales, and we are especially interested in few nanometer expansion, we adopted the setup shown in 

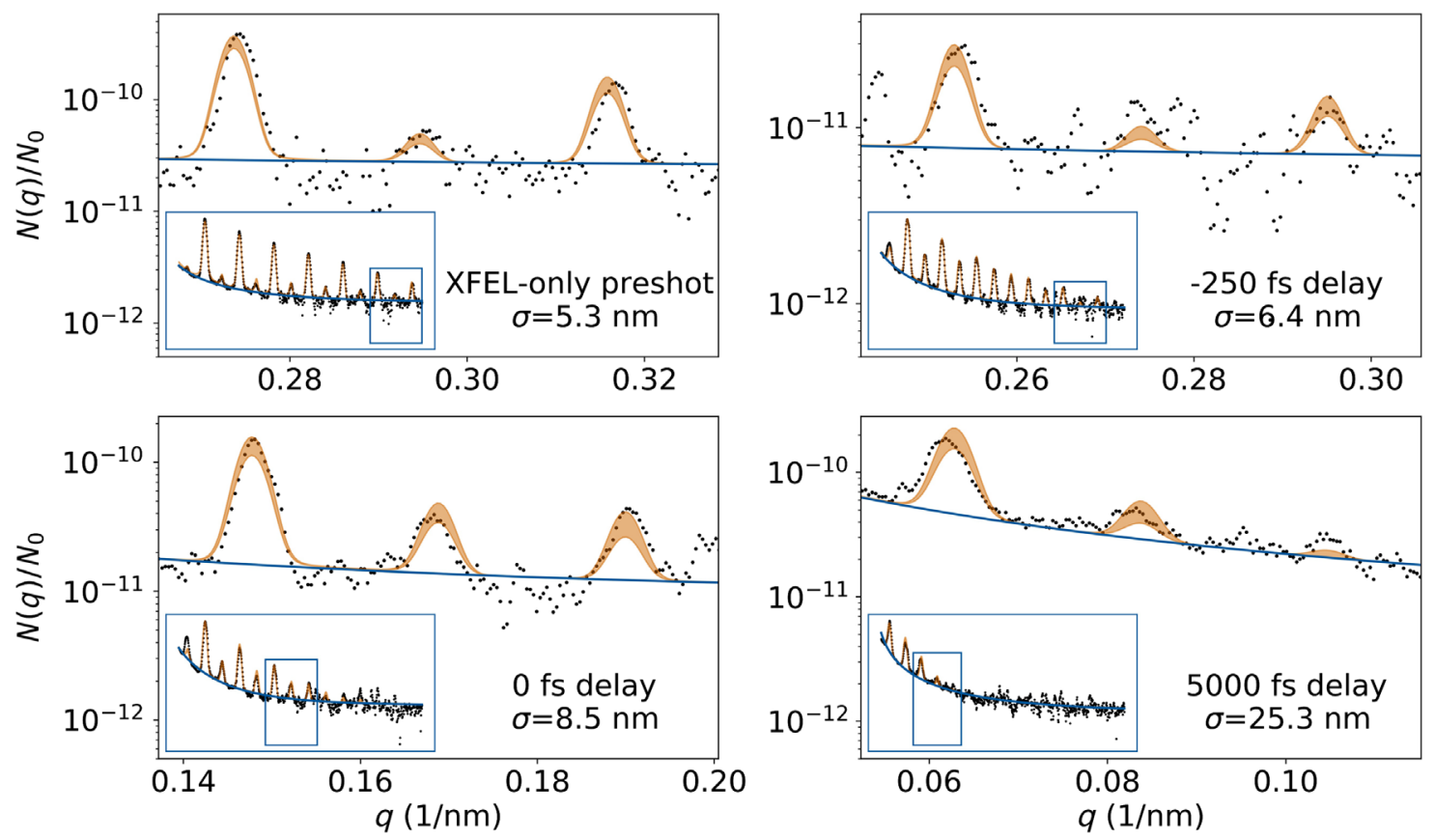

FIG. 7. Lineouts of scattered intensity normalized to the XFEL intensity (black points) taken along the $q$ direction perpendicular to the grating ridges, averaged over 20 pixels, the same as shown in Fig. 2. The reminiscent background was modeled by a power law (blue line) and subtracted prior to the fit. The regions shown are a zoom into the lineout as marked in the respective insets in order to make visible the shaded area for the relevant peaks at large $q$ values. They represent the range of possible fits with a $\chi<1.01 \chi$ min .

Fig. 1. Before fitting we subtract the electronic background (using separately recorded dark fields) and model the remaining x-ray background (e.g., from slit scattering at the XFEL cleaning apertures) with a power law. The individual heights of the peaks vary according to their phase in the single slit scattering function $S(q)$, which can reduce the fit sensitivity for $\sigma$ especially for large $\sigma$ where only a few maxima remain visible. This beat generates a correlation between $\sigma$ and $b / g$. It is possible to find different combinations of $\sigma$ and $b / g$ that lead to fits consistent with the data; this effect is included in the error bars in Fig. 3. The accuracy in determining $\sigma$ is given by the ability to measure the exponential decrease at large $q$ values. Thus, we achieve the highest accuracy, below a nanometer, for the smallest expansions at early times when many maxima are still visible. We determine the fit error by varying all fit parameters around the best-fit values and require $\chi^{2}$ to remain within $1 \%$ of the minimum $\chi_{\min }^{2}$ at the best fit, which corresponds to the requirement for the respective range of the variation of the estimator to cover the range of the experimental data variation. The projection of the resulting volume in parameter space onto the $\sigma$ axis gives the confidence interval for $\sigma$. This error level, as shown in Fig. 7, is a reasonable choice to describe the range of deviation between the data and the fit curve.

The accuracy of determining the expansion parameter $\sigma$ is demonstrated by comparison of the two XFEL-only preshots that we recorded before each optical laser shot, measuring the inherent smoothness due to the production process. On average, $\sigma_{0}$ inferred from the two preshots varies only by $0.7 \mathrm{~nm}$, which represents the total stochastic error and hence demonstrates the accuracy of the method within a chosen model for the plasma density profile.

For clarity, the error bars shown in Fig. 3 represent half of the $95 \%$ interval of the delay jitter distribution in the horizontal direction, and half of the $1 \%$ deviation interval around $\chi_{\min }^{2}$ in the vertical direction. For the data point marked in Fig. 3 [50], no fit error could be determined, but we still show the best-fit value for completeness.

\section{F. Numerical simulations}

Particle-in-cell simulations were performed using PICLS [67], a fully relativistic 2D code including field ionization and direct impact ionization [68]. The target was modeled as a planar Si foil with a 200-nm period grating of $100 \mathrm{~nm}$ height, with the ridges having an error-function-shaped density edge with $\sigma$ at the experimental average value of undriven gratings, determined by the XFEL preshots to be $5.4 \mathrm{~nm}$. We set 48 ions per cell, resulting in 672 electrons per cell at full ionization. The simulation box was set to $1 \lambda \times \lambda$, with 256 cells $/ \lambda$ and 256 time steps per laser period and periodic boundaries along the target surface. The laser was modeled as a spatially plane wave with a 80-fs-long Gaussian temporal profile, peak normalized field amplitude $a_{0}=0.38$ coming in from the left. We start the simulation $150 \mathrm{fs}$ prior to the arrival of the laser maximum on the target surface and follow the plasma evolution up to 2 ps later. At this time the plasma expansion was stagnating, the gradient 
staying constant within $\pm 1 \mathrm{~nm}$ over more than a picosecond. The shape of the edges remains error-function-like throughout the simulation. To extract the plasma expansion, we ignore the electron jets at times $>270 \mathrm{fs}$ and only project the density of the plasma bulk. The jets consistently occur in the simulations for a range of larger laser intensities (we performed additional simulations up to an order of magnitude higher intensity) and longer pulse durations (up factor of 2), always leading to a density pattern a few tens of nanometers in front of the surface following the preinscribed grating structure, shifted transversely by $g / 2$.

\section{DATA AVAILABILITY}

The data generated and used in this work is available under Creative Commons Attribution Share-Alike 4.0 licence on RODARE [69].

\section{ACKNOWLEDGMENTS}

Support by the Ion Beam Center, HZDR, is gratefully acknowledged. The authors thank L. Bischoff (HZDR) for his support in performing FIB cuts and electron microscopy of the target samples. This work was partially supported by DOE, Office of Science, Fusion Energy Science under FWP 100182. Use of the Linac Coherent Light Source (LCLS), SLAC National Accelerator Laboratory, is supported by the U.S. Department of Energy, Office of Science, Office of Basic Energy Sciences under Contract No. DE-AC0276SF00515. The experiments were performed at the Matter at Extreme Conditions (MEC) instrument of LCLS, supported by the DOE Office of Science, Fusion Energy Science under Contract No. SF00515. This work has also been supported by HIBEF and partially by Horizon 2020 LASERLAB-EUROPE/ LEPP (Contract No. 654148) and by the German Federal Ministry of Education and Research (BMBF) under Contract No. 03Z1O511. M. G. and M.Z. acknowledge support from the European Cluster of Advanced Laser Light Sources (EUCALL) project which has received funding from the European Union's Horizon 2020 research and innovation programme under Grant Agreement No. 654220. E. E. M. and C. R. acknowledge support from the Volkswagen Foundation.

[1] S. V. Bulanov and V.S. Khoroshkov, Feasibility of Using Laser Ion Accelerators in Proton Therapy, Plasma Phys. Rep. 28, 453 (2002).

[2] K. W. D. Ledingham, W. Galster, and R. Sauerbrey, LaserDriven Proton Oncology-A Unique New Cancer Therapy?, Br. J. Radiol. 80, 855 (2007).

[3] B. A. Remington, Modeling Astrophysical Phenomena in the Laboratory with Intense Lasers, Science 284, 1488 (1999).

[4] S. V. Bulanov, T. Zh. Esirkepov, M. Kando, J. Koga, K. Kondo, and G. Korn, On the Problems of Relativistic
Laboratory Astrophysics and Fundamental Physics with Super Powerful Lasers, Plasma Phys. Rep. 41, 1 (2015).

[5] M. Tabak, P. A. Norreys, V. T. Tikhonchuk, and K. A. Tanaka, Alternative Ignition Schemes in Inertial Confinement Fusion, Nucl. Fusion 54, 054001 (2014).

[6] M. Thévenet, A. Leblanc, S. Kahaly, H. Vincenti, A. Vernier, F. Quéré, and J. Faure, Vacuum Laser Acceleration of Relativistic Electrons Using Plasma Mirror Injectors, Nat. Phys. 12, 355 (2016).

[7] S. P. Hatchett et al., Electron, Photon, and Ion Beams from the Relativistic Interaction of Petawatt Laser Pulses with Solid Targets, Phys. Plasmas 7, 2076 (2000).

[8] J. Fuchs, P. Antici, E. D'Humières, E. Lefebvre, M. Borghesi, E. Brambrink, C. A. Cecchetti, M. Kaluza, V. Malka, M. Manclossi, S. Meyroneinc, P. Mora, J. Schreiber, T. Toncian, H. Pépin, and P. Audebert, Laser-Driven Proton Scaling Laws and New Paths towards Energy Increase, Nat. Phys. 2, 48 (2006).

[9] H. Schwoerer, S. Pfotenhauer, O. Jäckel, K.-U. Amthor, B. Liesfeld, W. Ziegler, R. Sauerbrey, K. W. D. Ledingham, and T. Esirkepov, Laser-Plasma Acceleration of QuasiMonoenergetic Protons from Microstructured Targets, Nature (London) 439, 445 (2006).

[10] A. Macchi, M. Borghesi, and M. Passoni, Ion Acceleration by Superintense Laser-Plasma Interaction, Rev. Mod. Phys. 85, 751 (2013).

[11] B. Dromey, M. Zepf, A. Gopal, K. Lancaster, M. S. Wei, K. Krushelnick, M. Tatarakis, N. Vakakis, S. Moustaizis, R. Kodama, M. Tampo, C. Stoeckl, R. Clarke, H. Habara, D. Neely, S. Karsch, and P. Norreys, High Harmonic Generation in the Relativistic Limit, Nat. Phys. 2, 456 (2006).

[12] C. Rödel et al., Harmonic Generation from Relativistic Plasma Surfaces in Ultrasteep Plasma Density Gradients, Phys. Rev. Lett. 109, 125002 (2012).

[13] S. Monchocé, S. Kahaly, A. Leblanc, L. Videau, P. Combis, F. Réau, D. Garzella, P. D'Oliveira, Ph. Martin, and F. Quéré, Optically Controlled Solid-Density Transient Plasma Gratings, Phys. Rev. Lett. 112, 145008 (2014).

[14] M. Roth et al., Bright Laser-Driven Neutron Source Based on the Relativistic Transparency of Solids, Phys. Rev. Lett. 110, 044802 (2013).

[15] M. Sherlock, E. G. Hill, R. G. Evans, S. J. Rose, and W. Rozmus, In-Depth Plasma-Wave Heating of Dense Plasma Irradiated by Short Laser Pulses, Phys. Rev. Lett. 113, 255001 (2014).

[16] T. Kluge, J. Metzkes, K. Zeil, M. Bussmann, U. Schramm, and T. E. Cowan, Two Surface Plasmon Decay of Plasma Oscillations, Phys. Plasmas 22, 064502 (2015).

[17] P. Leblanc and Y. Sentoku, Scaling of Resistive Guiding of Laser-Driven Fast-Electron Currents in Solid Targets, Phys. Rev. E 89, 023109 (2014).

[18] T. Kluge, M. Bussmann, L. Huang, J. Metzkes, T. E. Cowan, and U. Schramm, Current Scaling and Plasma Heating in Relativistic Laser-Solid Interaction, arXiv:1511.00497.

[19] Y. Sentoku, K. Mima, S.-i. Kojima, and H. Ruhl, Magnetic Instability by the Relativistic Laser Pulses in Overdense Plasmas, Phys. Plasmas 7, 689 (2000).

[20] M. Borghesi et al., in Ion source development and radiobiology applications within the LIBRA project, in edited by 
K. W. D. Ledingham, W. P. Leemans, E. Esarey, S. M. Hooker, K. Spohr, and P. McKenna, SPIE Proceedings Vol. 8079 (SPIE-International Society for Optical Engineering, Bellingham, WA, 2011), p. 80791E.

[21] C. A. J. Palmer et al., Rayleigh-Taylor Instability of an Ultrathin Foil Accelerated by the Radiation Pressure of an Intense Laser, Phys. Rev. Lett. 108, 225002 (2012).

[22] J. Metzkes, T. Kluge, K. Zeil, M. Bussmann, S. D. Kraft, T. E. Cowan, and U. Schramm, Experimental Observation of Transverse Modulations in Laser-Driven Proton Beams, New J. Phys. 16, 023008 (2014).

[23] A. Sgattoni, S. Sinigardi, L. Fedeli, F. Pegoraro, and A. Macchi, Laser-Driven Rayleigh-Taylor Instability: Plasmonic Effects and Three-Dimensional Structures, Phys. Rev. E 91, 013106 (2015).

[24] S. Göde, C. Rödel, K. Zeil, R. Mishra, M. Gauthier, F.-E. Brack, T. Kluge, M. J. MacDonald, J. Metzkes, L. Obst, M. Rehwald, C. Ruyer, H.-P. Schlenvoigt, W. Schumaker, P. Sommer, T. E. Cowan, U. Schramm, S. Glenzer, and F. Fiuza, Relativistic Electron Streaming Instabilities Modulate Proton Beams Accelerated in Laser-Plasma Interactions, Phys. Rev. Lett. 118, 194801 (2017).

[25] P. Mora, Plasma Expansion into a Vacuum, Phys. Rev. Lett. 90, 185002 (2003).

[26] P. Mora, Thin-Foil Expansion into a Vacuum, Phys. Rev. E 72, 056401 (2005).

[27] J. Schreiber, F. Bell, F. Grüner, U. Schramm, M. Geissler, M. Schnürer, S. Ter-Avetisyan, B. M. Hegelich, J. Cobble, E. Brambrink, J. Fuchs, P. Audebert, and D. Habs, Analytical Model for Ion Acceleration by High-Intensity Laser Pulses, Phys. Rev. Lett. 97, 045005 (2006).

[28] Y. Ping, R. Shepherd, B. F. Lasinski, M. Tabak, H. Chen, H. K. Chung, K. B. Fournier, S. B. Hansen, A. Kemp, D. A. Liedahl, K. Widmann, S. C. Wilks, W. Rozmus, and M. Sherlock, Absorption of Short Laser Pulses on Solid Targets in the Ultrarelativistic Regime, Phys. Rev. Lett. 100, 085004 (2008).

[29] M. Kaluza, J. Schreiber, M. I. K. Santala, G. D. Tsakiris, K. Eidmann, J. Meyer-ter-Vehn, and K. J. Witte, Influence of the Laser Prepulse on Proton Acceleration in Thin-Foil Experiments, Phys. Rev. Lett. 93, 045003 (2004).

[30] S. Kahaly, S. Monchocé, H. Vincenti, T. Dzelzainis, B. Dromey, M. Zepf, Ph. Martin, and F. Quéré, Direct Observation of Density-Gradient Effects in Harmonic Generation from Plasma Mirrors, Phys. Rev. Lett. 110, 175001 (2013).

[31] S. Kahaly, S. K. Yadav, W. M. Wang, S. Sengupta, Z. M. Sheng, A. Das, P. K. Kaw, and G. Ravindra Kumar, NearComplete Absorption of Intense, Ultrashort Laser Light by Sub- $\lambda$ Gratings, Phys. Rev. Lett. 101, 145001 (2008).

[32] T. Ceccotti et al., Evidence of Resonant Surface-Wave Excitation in the Relativistic Regime through Measurements of Proton Acceleration from Grating Targets, Phys. Rev. Lett. 111, 185001 (2013).

[33] M. Cerchez, A. L. Giesecke, C. Peth, M. Toncian, B. Albertazzi, J. Fuchs, O. Willi, and T. Toncian, Generation of Laser-Driven Higher Harmonics from Grating Targets, Phys. Rev. Lett. 110, 065003 (2013).

[34] J. P. Geindre, A. Mysyrowicz, A. Dos Santos, P. Audebert, A. Rousse, G. Hamoniaux, A. Antonetti, F. Falliès, and J. C.
Gauthier, Frequency-Domain Interferometer for Measuring the Phase and Amplitude of a Femtosecond Pulse Probing a Laser-Produced Plasma, Opt. Lett. 19, 1997 (1994).

[35] S. Mondal, A. D. Lad, S. Ahmed, V. Narayanan, J. Pasley, P. P. Rajeev, A. P. L. Robinson, and G. R. Kumar, Doppler Spectrometry for Ultrafast Temporal Mapping of Density Dynamics in Laser-Induced Plasmas, Phys. Rev. Lett. 105, 105002 (2010).

[36] T. Kluge, C. Gutt, L. G. Huang, J. Metzkes, U. Schramm, M. Bussmann, and T.E. Cowan, Using X-Ray FreeElectron Lasers for Probing of Complex Interaction Dynamics of Ultra-Intense Lasers with Solid Matter, Phys. Plasmas 21, 033110 (2014).

[37] D. Milathianaki, S. Boutet, G. J. Williams, A. Higginbotham, D. Ratner, A. E. Gleason, M. Messerschmidt, M. M. Seibert, D. C. Swift, P. Hering, J. Robinson, W. E. White, and J. S. Wark, Femtosecond Visualization of Lattice Dynamics in Shock-Compressed Matter, Science 342, 220 (2013).

[38] M. Gauthier et al., New Experimental Platform to Study High Density Laser-Compressed Matter, Rev. Sci. Instrum. 85, $11 \mathrm{E} 616$ (2014).

[39] M. P. Olbinado, V. Cantelli, O. Mathon, S. Pascarelli, J. Grenzer, A. Pelka, M. Roedel, I. Prencipe, A. L. Garcia, U. Helbig, D. Kraus, U. Schramm, T. Cowan, M. Scheel, P. Pradel, T. De Resseguier, and A. Rack, Ultra High-Speed $X$-Ray Imaging of Laser-Driven Shock Compression Using Synchrotron Light, J. Phys. D 51, 055601 (2018).

[40] F. Seiboth, A. Schropp, R. Hoppe, V. Meier, J. Patommel, H. J. Lee, B. Nagler, E. C. Galtier, B. Arnold, U. Zastrau, J. B. Hastings, D. Nilsson, F. Uhlén, U. Vogt, H. M. Hertz, and C. G. Schroer, Focusing XFEL SASE Pulses by Rotationally Parabolic Refractive X-Ray Lenses, J. Phys. Conf. Ser. 499, 012004 (2014).

[41] A. Schropp, R. Hoppe, V. Meier, J. Patommel, F. Seiboth, Y. Ping, D. G. Hicks, M. A. Beckwith, G. W. Collins, A. Higginbotham, J. S. Wark, H. J. Lee, B. Nagler, E. C. Galtier, B. Arnold, U. Zastrau, J. B. Hastings, and C. G. Schroer, Imaging Shock Waves in Diamond with Both High Temporal and Spatial Resolution at an XFEL, Sci. Rep. 5, 11089 (2015).

[42] F. Seiboth et al., Perfect X-Ray Focusing via Fitting Corrective Glasses to Aberrated Optics, Nat. Commun. 8, 14623 (2017).

[43] O. Glatter and O. Kratky, Acta Polymerica (Academic Press Inc. Ltd., London, 1982); W. C. Michels, A. L. Patterson, and Roald K. Wangsness, Elements of Modern Physics, Am. J. Phys. 20, 58 (1952).

[44] H. N. Chapman et al., Femtosecond Diffractive Imaging with a Soft-X-Ray Free-Electron Laser, Nat. Phys. 2, 839 (2006).

[45] H. N. Chapman et al., Femtosecond Time-Delay X-Ray Holography, Nature (London) 448, 676 (2007).

[46] T. Gorkhover et al., Femtosecond and Nanometre Visualization of Structural Dynamics in Superheated Nanoparticles, Nat. Photonics 10, 93 (2016).

[47] M Nakatsutsumi, K. Appel, G. Priebe, I. Thorpe, A. Pelka, B. Muller, and Th. Tschentscher, Technical Design Report: Scientific Instrument High Energy Density Physics (HED) (European X-Ray Free-Electron Laser Facility $\mathrm{GmbH}$, Hamburg, Germany, 2014). 
[48] B. Nagler et al., The Matter in Extreme Conditions Instrument at the Linac Coherent Light Source, J. Synchrotron Radiat. 22, 520 (2015).

[49] L. B. Fletcher et al., Ultrabright X-Ray Laser Scattering for Dynamic Warm Dense Matter Physics, Nat. Photonics 9, 274 (2015).

[50] M. R. Bionta, H. T. Lemke, J. P. Cryan, J. M. Glownia, C. Bostedt, M. Cammarata, J.-C. Castagna, Y. Ding, D. M. Fritz, A. R. Fry, J. Krzywinski, M. Messerschmidt, S. Schorb, M. L. Swiggers, and R. N. Coffee, Spectral Encoding of X-Ray/Optical Relative Delay, Opt. Express 19, 21855 (2011).

[51] M. Harmand, R. Coffee, M. R. Bionta, M. Chollet, D. French, D. Zhu, D. M. Fritz, H. T. Lemke, N. Medvedev, B. Ziaja, S. Toleikis, and M. Cammarata, Achieving FewFemtosecond Time-Sorting at Hard X-Ray Free-Electron Lasers, Nat. Photonics 7, 215 (2013).

[52] One target showed a somewhat larger initial ridge sharpness of $\sigma_{0}=11.3 \mathrm{~nm}$, marked by the arrow in Fig. 3 .

[53] K. Quinn, L. Romagnani, B. Ramakrishna, G. Sarri, M. E. Dieckmann, P. A. Wilson, J. Fuchs, L. Lancia, A. Pipahl, T. Toncian, O. Willi, R. J. Clarke, M. Notley, A. Macchi, and M. Borghesi, Weibel-Induced Filamentation during an Ultrafast Laser-Driven Plasma Expansion, Phys. Rev. Lett. 108, 135001 (2012).

[54] T. Kluge et al., Nanometer-Scale Characterization of LaserDriven Compression, Shocks, and Phase Transitions, by $X$-Ray Scattering Using Free Electron Lasers, Phys. Plasmas 24, 102709 (2017).

[55] C. Gerthsen and H. Vogel, Gerthsen Physik, 17th ed. (Springer-Lehrbuch, Berlin, 1993), p. 489f.

[56] E. W. Ng and M. Geller, A Table of Integrals of the Error Functions, J. Res. Natl. Bur. Stand. 73B, 1 (1969).

[57] See Supplemental Material at http://link.aps.org/ supplemental/10.1103/PhysRevX.8.031068 for details on Fig. 4 and movie of the temporal evolution of the simulated electron density.

[58] F. Bencivenga, R. Cucini, F. Capotondi, A. Battistoni, R. Mincigrucci, E. Giangrisostomi, A. Gessini, M. Manfredda, I. P. Nikolov, E. Pedersoli, E. Principi, C. Svetina, P. Parisse, F. Casolari, M. B. Danailov, M. Kiskinova, and C. Masciovecchio, Four-Wave Mixing Experiments with Extreme Ultraviolet Transient Gratings, Nature (London) 520, 205 (2015).
[59] S. H. Glenzer et al., Symmetric Inertial Confinement Fusion Implosions at Ultra-High Laser Energies, Science 327, 1228 (2010).

[60] I. Prencipe et al., Targets for High Repetition Rate Laser Facilities: Needs, Challenges and Perspectives, High Power Laser Sci. Eng. 5, e17 (2017).

[61] A. Maksimchuk, S. Gu, K. Flippo, D. Umstadter, and V. Bychenkov, Forward Ion Acceleration in Thin Films Driven by a High-Intensity Laser, Phys. Rev. Lett. 84, 4108 (2000).

[62] S. C. Wilks, A. B. Langdon, T.E. Cowan, M. Roth, M. Singh, S. Hatchett, M. H. Key, D. Pennington, A. MacKinnon, and R. A. Snavely, Energetic Proton Generation in Ultra-Intense Laser-Solid Interactions, Phys. Plasmas 8, 542 (2001).

[63] L. G. Huang, M. Bussmann, T. Kluge, A. L. Lei, W. Yu, and T. E. Cowan, Ion Heating Dynamics in Solid Buried Layer Targets Irradiated by Ultra-Short Intense Laser Pulses, Phys. Plasmas 20, 093109 (2013).

[64] L. G. Huang, T. Kluge, and T. E. Cowan, Dynamics of Bulk Electron Heating and Ionization in Solid Density Plasmas Driven by Ultra-Short Relativistic Laser Pulses, Phys. Plasmas 23, 063112 (2016).

[65] T. Kluge, M. Bussmann, H.-K. Chung, C. Gutt, L. G. Huang, M. Zacharias, U. Schramm, T.E. Cowan, M. Bussmann, H.-K. Chung, C. Gutt, L. G. Huang, M. Zacharias, M. Bussmann, U. Schramm, and T. E. Cowan, Nanoscale Femtosecond Imaging of Transient Hot Solid Density Plasmas with Elemental and Charge State Sensitivity Using Resonant Coherent Diffraction, Phys. Plasmas 23, 033103 (2016).

[66] T. Oksenhendler, P. Bizouard, O. Albert, S. Bock, and U. Schramm, High Dynamic, High Resolution and Wide Range Single Shot Temporal Pulse Contrast Measurement, Opt. Express 25, 12588 (2017).

[67] Y. Sentoku and A. J. Kemp, Numerical Methods for Particle Simulations at Extreme Densities and Temperatures: Weighted Particles, Relativistic Collisions and Reduced Currents, J. Comput. Phys. 227, 6846 (2008).

[68] R. Mishra, P. Leblanc, Y. Sentoku, M. S. Wei, and F. N. Beg, Collisional Particle-in-Cell Modeling for Energy Transport Accompanied by Atomic Processes in Dense Plasmas, Phys. Plasmas 20, 072704 (2013).

[69] T. Kluge et al., Data for publication, RODARE, DOI: 10.14278/rodare.24. 